\title{
Lohnunterschiede im Spannungsfeld von sozialer Herkunft und individueller Bildung
}

Eine vergleichende Analyse für Deutschland und die Schweiz ${ }^{1}$

\section{Von Bettina Isengard}

Zusammenfassung: Die Humankapitaltheorie ist der dominierende Ansatz, wenn es darum geht, individuelle Lohnunterschiede zu erklären. Demnach ist der verfügbare Bestand an Humankapital allgemein und insbesondere das Bildungsniveau das zentrale Merkmal für den individuellen Arbeitsmarkterfolg bzw. die Lohnhöhe. Entsprechend können Unterschiede in den Erwerbseinkommen auf Basis von Produktivitätsunterschieden, die sich durch ungleiche Bildungsqualifikationen ergeben, gerechtfertigt werden. Aber gleichzeitig postuliert die Statuszuweisungstheorie einen engen Zusammenhang zwischen der sozialen Herkunft, der individuellen Bildung und dem persönlichen Arbeitsmarkterfolg. Nach diesem Ansatz beeinflusst der Sozialstatus des Elternhauses die formale Bildungsqualifikation der Kinder, die wiederum den beruflichen Status und somit die Lohnhöhe determiniert. Parallel dazu kann die Herkunft aber auch einen direkten Effekt auf die Erwerbseinkommen haben. Vor diesem Hintergrund untersucht der Beitrag die Bedeutung der sozialen Herkunft für den Bildungs- und insbesondere Arbeitsmarkterfolg in Deutschland und der Schweiz. Dabei zeigt sich, dass sich beide Länder insgesamt sehr ähnlich sind, was den Zusammenhang zwischen sozialer Herkunft, Bildung und Einkommen angeht.

\section{Einleitende Bemerkungen}

In den modernen westlichen Gesellschaften gehören Wohlstand und die gerechte Verteilung von Lebenschancen zu den grundlegenden gesellschaftspolitischen Zielen. Das verfügbare Einkommen ist dabei der zentrale Gradmesser für diese Ziele und resultiert aus einem mehrstufigen Verteilungsprozess, an dessen Anfang die Erwerbseinkommen stehen. Diese sind ein wichtiger Indikator der ungleichen Verteilung, weil sich soziale Ungleichheit in modernen Volkswirtschaften für den Großteil der Individuen über den Arbeitsmarkt reguliert bzw. durch den dortigen (Nicht-)Erfolg manifestiert und auch Personen, die nicht direkt am Erwerbsleben teilnehmen und kein eigenes Erwerbseinkommen beziehen, indirekt vom Arbeitsmarkt abhängig sind (vgl. z.B. Atkinson 1983: 97; Szydlik 1993: 11; Hirschel 2004: 69). Lohnunterschiede wiederum können in meritokratischen Gesellschaften auf Basis des Leistungsprinzips gerechtfertigt werden und individuelle Leistungsunterschiede, die auf dem Arbeitsmarkt zumeist über die formale Bildungsqualifikation abgebildet werden, fungieren als Legitimationsgrundlage (vgl. Davis / Moore [1945] 1967). Das Leistungsprinzip, das seinen Ursprung im frühindustriellen Wirtschaftsbürgertum Europas hat, postuliert, dass einhergehend mit der Auflösung der Ständegesellschaften das Geburtsprinzip, die soziale Herkunft, an Bedeutung verloren und sich der individualisierte Leistungsgedanke zunehmend durchgesetzt hat (für einen Überblick vgl. Hradil 2001: 417).

Allerdings deuten empirische Untersuchungen darauf hin, dass die Prozesse, die ungleiche Einkommensergebnisse erzeugen, sehr komplex sind, sich nicht allein durch individuelle Leistungs- bzw. Bildungsunterschiede erklären lassen und der Mythos „Reich durch Leistung“ in der Realität nur bedingt zutrifft. Ein klassisches Beispiel ist die Untersuchung des Soziolo-

1 Ich danke Walter Müller und Gert G. Wagner, die mich im Rahmen meiner Dissertation, aus der dieser Aufsatz hervorgegangen ist, betreut und unterstützt haben, Martin Kroh und Thorsten Schneider für zahlreiche gute Anmerkungen und Kommentare sowie einem anonymen Gutachter für hilfreiche Anregungen.

Soziale Welt 60 (2009), S. $27-45$ 
gen Jencks, der für die USA zu Beginn der 1970er Jahre herausfand, dass Einkommen und Sozialstatus zwar von dem individuellen Bildungsniveau, der zertifizierten Leistungsfähigkeit, abhängen, dieser Einfluss aber eher mäßig ist (vgl. Jencks et al. 1972). Auch für andere Länder kann dieses „Phänomen“ bereits in den 70er Jahren bestätigt werden (vgl. Müller / Mayer 1976; Girod 1977). Neben der individuellen Leistung bzw. Bildung spielt bei der Verteilung von ökonomischen Ressourcen offenbar nach wie vor die „Gnade der Geburt“, die soziale Herkunft, eine wichtige Rolle. Dadurch wird jedoch die empirische Gültigkeit des Leistungsprinzips eingeschränkt und das meritokratische Prinzip konterkariert. Individuelle Lohnunterschiede können zwar als gerecht erachtet werden, wenn sie auf Bildungsunterschiede zurückzuführen sind, doch dazu müssten die Ausgangsbedingungen beim Bildungserwerb für alle gleich sein. Die Chancengleichheit existiert jedoch häufig nur auf dem Papier und wäre erst gegeben, wenn „der Zugang zu Belohnungen [...] nur durch eigene Leistung und nicht durch Glück, List oder Herkunft geregelt wird“" (Hondrich 1984: 275).

Sowohl Deutschland als auch die Schweiz sind individualisierte Leistungsgesellschaften, die dadurch gekennzeichnet sind, dass sie dem meritokratischen Gedanken folgend offene Gesellschaften sind, in denen sich Leistungsunterschiede in der Lohnhöhe niederschlagen sollten. Der deutsch-schweizerische Vergleich bietet sich an, da sich beide Nationen in der Organisationsstruktur der Bildungssysteme sehr ähnlich sind, was zur Folge hat, dass hier nach wie vor soziale Herkunftseffekte den individuellen Bildungserfolg mitbestimmen. Dagegen sind die Arbeitsmarktstrukturen und wohlfahrtsstaatlichen Arrangements in beiden Ländern unterschiedlich und das hat unmittelbaren Einfluss auf das Leistungsverständnis sowie die Rolle von Individuum und Familie, was sich in Unterschieden im Einfluss der Bildung und auch der sozialen Herkunft auf Einkommen niederschlagen sollte.

Ziel dieses Beitrags ist es vor diesem Hintergrund, den Zusammenhang zwischen der sozialen Herkunft, individueller Bildung und Lohnunterschieden zu analysieren. Dazu wird im folgenden Abschnitt zunächst ein Überblick über die relevanten Theorien und ihre empirische Evidenz gegeben. Anschließend (Abschnitt 3) werden die Forschungshypothesen sowie die Modellspezifikationen dargelegt. Abschnitt 4 beschreibt die verwendeten Datensätze und die Operationalisierung der Variablen. Die empirischen Ergebnisse werden in Abschnitt 5 dargestellt und diskutiert. Abschließende Bemerkungen sowie sozialpolitische Implikationen auf Basis der Ergebnisse werden im letzten Abschnitt (6) erläutert.

\section{Theoretischer Hintergrund und empirische Evidenz}

Wenn es darum geht individuelle Erwerbseinkommensunterschiede zu erklären, dann gibt es in der gängigen Literatur zwei theoretische Hauptrichtungen, die zwischen individuellen und strukturellen Determinanten differenzieren. Während die individuellen Theorien insbesondere auf die persönlichen Charakteristika der Empfänger von Erwerbseinkommen abzielen und diese für Lohnunterschiede verantwortlich machen, rücken die strukturellen Ansätze dagegen die vorherrschenden Arbeitsstrukturen in den Vordergrund. ${ }^{2}$ Atkinson (1983: 97ff) teilt die individuellen Bestimmungsmerkmale von Einkommen nach drei Hauptcharakteristika ein und unterscheidet zwischen (a) angeborenen, genetisch bedingten Fähigkeiten und Talenten, ${ }^{3}$ (b)

2 Der folgende Beitrag konzentriert sich nur auf die individuellen Ursachen von Lohnunterschieden. Allerdings zeigen weiterführende - hier nicht dargestellte - Analysen, dass die Arbeitsmarktstrukturen zwar in beiden Ländern einen wichtigen Einfluss auf die Erwerbseinkommenshöhe haben, die individuellen Faktoren, die hier im Mittelpunkt des Interesses stehen, jedoch in ihrer Erklärungskraft nicht beeinflussen. Deswegen wird hier aus Gründen der Übersichtlichkeit auf eine Darstellung verzichtet.

3 Neuere Untersuchungen schreiben auch anderen, auf den ersten Blick abwegigeren, teilweise angeborenen Indikatoren wie der Körpergröße (Heineck 2005) oder dem Gewicht (Gautschi / Hangartner 2006) eine zentrale Rolle zu. 
erworbenen und zumeist durch (Aus-)Bildung angeeigneten Kenntnissen und (c) von der sozialen Herkunft abhängigen Fertigkeiten. ${ }^{4}$ Aufgrund von datentechnischen Beschränkungen ist die Berücksichtigung angeborener Fähigkeiten in den Analysen nicht möglich. Deswegen erfolgt hier eine Konzentration auf die beiden anderen Indikatoren, d.h. Bildung und soziale Herkunft. Um die Zusammenhangsstrukturen zwischen den Merkmalen Herkunft, Bildung und Einkommen zu analysieren, kann insbesondere auf zwei zentrale Erklärungsansätze zurückgegriffen werden: die Humankapitaltheorie sowie die Statuszuweisungstheorie.

Die Humankapitaltheorie (Mincer 1970; Becker 1964) geht davon aus, dass Individuen am Arbeitsmarkt auf Basis ihrer Produktivität entlohnt werden, die ihrerseits wiederum von der jeweiligen Qualifikation bzw. dem Humankapitalbestand abhängt. Dahinter steht die Vermutung, dass mit besserer (Aus-)Bildung die Produktivität höher ist und somit höhere Löhne gezahlt werden. Das Schooling-Modell von Mincer (1970: 7), das Basismodell der Humankapitaltheorie, nimmt beispielsweise an, dass die Dauer der schulischen Ausbildung und die Berufserfahrung die Arbeitsproduktivität erhöhen und somit die individuelle Lohnhöhe bestimmen. Neuere humankapitaltheoretische Ansätze (vgl. Card 1995; Bowles et al. 2001 a) berücksichtigen neben den klassischen Humankapitalindikatoren wie Bildungszeit und Berufserfahrung explizit soziodemografische Merkmale sowie die soziale Herkunft. Insgesamt ist die empirische Relevanz der humankapitaltheoretischen Ansätze groß. So zeigen zahlreiche empirische Studien zu diesem Thema, dass ein Großteil der Einkommensungleichheiten auf individuelle Produktivitätsunterschiede zurückzuführen ist und das Bildungssystem somit in offenen Leistungsgesellschaften eine entscheidende Funktion bei der Weichenstellung für Ansehen und gesellschaftliche Privilegien hat (für einen umfassenden Überblick über den allgemeinen Forschungsstand vgl. Heckman et al. 2003; für Deutschland Bellmann et al. 1984; Schwarze 1991). ${ }^{5}$ Allerdings begrenzen verschiedene Merkmale wie beispielsweise die persönliche Begabung, die materielle Ausstattung des Elternhauses oder auch die unterschiedlichen Zugangsmöglichkeiten zu Bildungseinrichtungen die Ausbildungsmöglichkeiten und somit die Chancengleichheit (Mincer 1976: 149).

Denn wie Studien zu Bildungsentscheidungen zeigen, ist die Wahl eines Bildungsweges zum Großteil schichtspezifisch vorherbestimmt (vgl. Saterdag / Stegmann 1980; Erikson / Jonsson 1996) und verschiedene Studien für Deutschland und die Schweiz finden heraus, dass in diesen Ländern nach wie vor Unterschiede beim Bildungserwerb bestehen, die nach sozialen Schichten variieren (vgl. für Deutschland z.B. Schimpl-Neimanns 2000; Wolff 1997: 247ff; für die Schweiz Buchmann / Sacchi 1998; Buchmann / Charles 1993; sowie international Prenzel et al. 2004; Shavit / Blossfeld 1993). Die Bildungsverteilung spiegelt also nach wie vor die Privilegienverteilung wider, da die schulische Selektion von der sozialen Herkunft abhängt (Bourdieu / Passeron 1971). Der Zusammenhang zwischen Bildung und Einkommen ist somit nicht nur auf individuelle Produktivitätsunterschiede zurückzuführen, sondern verschleiert die dahinter liegenden tatsächlichen Wirkungszusammenhänge, die insbesondere von der sozialen Herkunft ausgehen.

4 Diese drei Charakteristika sind allerdings in der Realität nicht unabhängig voneinander, da die individuellen Fähigkeiten und (formalen) Qualifikationen, die ungleiche Einkommen bedingen, auf einem Zusammenspiel aller drei Merkmale basieren.

5 Demgegenüber gibt es aber auch die Ansicht, dass die Bildung für den Arbeitsmarkterfolg weniger bedeutsam ist als bislang angenommen, da Arbeitgeber bei Einstellungsentscheidungen offenbar der Bildungsqualifikation weniger Gewicht beimessen und andere Eigenschaften der Bewerber in den Vordergrund treten (vgl. Jackson et al. 2005). Entsprechend gewinnt in jüngster Zeit auch ein Forschungsstrang zunehmend an Bedeutung, der sich mit der Frage nach dem Einfluss nicht-kognitiver Eigenschaften, so genannter „Soft Skills“, beschäftigt. So zeigen einige Untersuchungen, dass neben den kognitiven Fähigkeiten, der Bildung, und der sozialen Herkunft auch Merkmale der Persönlichkeit für die Erklärung von Einkommen bedeutsam sind (vgl. dazu z.B. Bowles et al. 2001 b; Borghans et al. 2008). 
Deswegen kann die Statuszuweisungstheorie herangezogen werden, um den Zusammenhang zwischen der Herkunft, der Bildung sowie dem (späteren) Einkommenserfolg zu beschreiben. Diese Theorie entstammt der soziologischen Mobilitätsforschung und ist insbesondere durch die Untersuchung über „The American Occupational Structure“ von Blau und Duncan (1967) bekannt geworden. ${ }^{6}$ Nach diesem Ansatz ist der berufliche Status, den ein Individuum zu einem bestimmten Zeitpunkt im Lebenslauf inne hat, vor allem auf soziale Herkunft, Intelligenz, Fähigkeiten und schulische Bildung zurückzuführen, also insgesamt auf Merkmale, die vor dem Eintritt in das Beschäftigungssystem angesiedelt sind. Entsprechend der Theorie kann für Ungleichheiten im Allgemeinen und Lohnunterschiede im Spezifischen insbesondere die soziale Herkunft verantwortlich gemacht werden (vgl. z.B. Bowles 1972; Bowles et al. 2001 a; Dustmann 2004). Denn diese begrenzt die (schulischen und beruflichen) Wahlmöglichkeiten von Individuen teilweise erheblich und dadurch auch das spätere potentielle Einkommen. Üblicherweise werden hierbei mögliche strukturbedingte Einflüsse als konstant angenommen. Im Mittelpunkt stehen bei diesem Ansatz zentrale Merkmale des Elternhauses wie z.B. die Bildung oder der Beruf der Eltern, aber auch die finanzielle Situation in der Kindheit. ${ }^{7}$

Trotz der bestehenden Einigkeit in der sozialwissenschaftlichen Forschung über die grundlegende Annahme, dass Herkunft und Bildung die Lebenschancen der Individuen nachhaltig beeinflussen, können bezüglich der genauen Wirkungsmechanismen unterschiedliche kausale Annahmen getroffen werden. Dabei werden insbesondere vier zentrale Modelle unterschieden (vgl. Schnapp 1997: 84), die im Folgenden kurz dargestellt und erläutert werden (vgl. Abbildung 1).

\section{Abb. 1: Zum Zusammenhang zwischen sozialer Herkunft, Bildung und Einkommen}

Modell 1

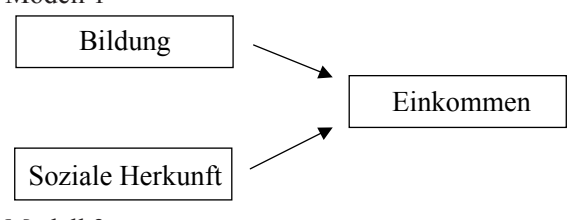

Modell 3

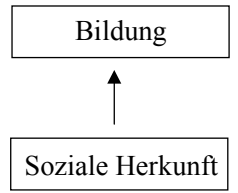

Modell 2

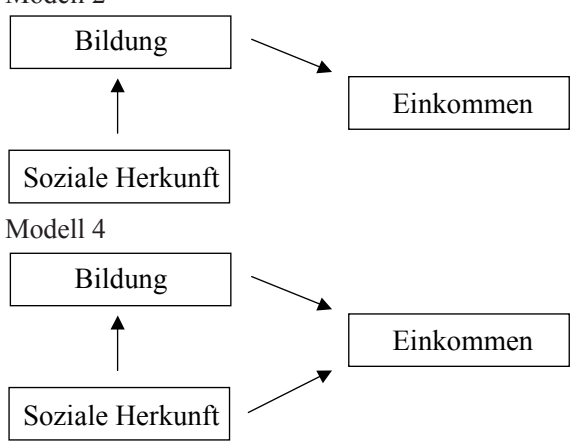

Quelle: eigene Darstellung in Anlehnung an Schnapp (1997: 84).

Nach Modell 1 wird das Einkommen eines Individuums sowohl durch die (eigene) Bildung als auch durch die soziale Herkunft beeinflusst, wobei allerdings zwischen diesen Einflussfaktoren kein Zusammenhang besteht. Diese Annahme erscheint jedoch vor dem Hintergrund der bisherigen Forschung nicht realistisch, da zahlreiche empirische Befunde einen Zusammenhang zwischen der sozialen Herkunft und der formalen Bildungsqualifikation feststellen

6 Das „Wisconsin Model of Status Attainment“ modifiziert das Blau-Duncan-Modell um sozialpsychologische Aspekte (Sewell et al. 1969). Dabei wird angenommen, dass der Herkunftseffekt auf die Bildung (und den beruflichen Erfolg) durch Einflüsse von Bezugsgruppen und die Bildungsaspiration bzw. berufliche Aspirationen vermittelt wird (vgl. für Deutschland Müller 1972).

7 Ob der Einfluss des Vaters oder der Mutter dabei wichtiger ist, darüber wird in der Forschung kontrovers diskutiert (vgl. z.B. Schneider 2004; Ermisch / Francesconi 2001; Gang / Zimmermann 2000). 
(für Deutschland Schimpl-Neimanns 2000; für die Schweiz Ramseier / Brühwiler 2003). Modell 2 stellt die Wirkungszusammenhänge derart dar, dass die soziale Herkunft das Bildungsniveau und dieses wiederum das (eigene) Einkommen beeinflusst. Nach diesem theoretischen Modell hat die soziale Herkunft keinen direkten Einfluss auf das individuelle Einkommen, sondern nur einen indirekten Effekt, der über die Bildung vermittelt wird. Entsprechend Modell 3 hat die soziale Herkunft sowohl einen direkten Einfluss auf die (eigene) Bildung als auch auf das Einkommen. Im Gegensatz zu den beiden vorhergehenden Modellen hat hier jedoch die Bildung keinen Einfluss auf das Einkommen. Dieses Modell steht damit in Widerspruch zu den humankapitaltheoretischen Theorien, die der Bildung eine zentrale Rolle bei der Einkommenserzielung beimessen (vgl. Becker 1964; Mincer 1974) und widerspricht zusätzlich auch der Leistungsideologie in meritokratischen Gesellschaften sowie zahlreichen empirischen Ergebnissen. Modell 4 schließlich postuliert wie Modell 3, dass die soziale Herkunft sowohl die Bildung als auch das Einkommen direkt beeinflusst. Zusätzlich wirkt ein indirekter Herkunftseffekt auf das Einkommen, der über die Bildung vermittelt wird. Empirisch findet dieses Modell z.B. bei der Vergabe von Berufspositionen Unterstützung (Hartmann 2002) und am Beispiel zum Zugang zu Führungspositionen im Zusammenhang mit sozialen Herkunftseffekten und Promotionen zeigen Hartmann und Kopp (2001: 436), dass ,die soziale Herkunft [...] einen wesentlichen eigenständigen Faktor der sozialen Auslese“ darstellt.

\section{Hypothesen und Modellspezifikationen}

Auf Basis der humankapitaltheoretischen Überlegungen, der Statuszuweisungstheorie sowie bisheriger empirischer Befunde können verschiedene Forschungshypothesen aufgestellt werden, die im Folgenden empirisch untersucht werden. Vor dem Hintergrund der aktuellen PISABefunde und anderer Studien kann angenommen werden, dass in Deutschland und der Schweiz der individuelle Bildungserfolg stark von der sozialen Herkunft abhängt (Hypothese 1), aber auch, dass dieser Zusammenhang in Deutschland stärker ist als in der Schweiz (Hypothese 2). Das individuelle Bildungsniveau seinerseits beeinflusst ebenso wie die Ausstattung an spezifischem Humankapital nach humankapitaltheoretischen Überlegungen sowohl in Deutschland als auch der Schweiz den Arbeitsmarkterfolg, der über die Lohnhöhe gemessen wird (Hypothese 3). Gleichzeitig kann auch die Statuszuweisungstheorie für die Erklärung von Lohnunterschieden herangezogen werden. Demnach beeinflusst auch die soziale Herkunft die Lohnhöhe, wobei dieser Einfluss einerseits indirekter Natur sein kann und über die Bildung vermittelt wird, sowie andererseits auch direkt wirken kann (Hypothese 4).

Neben Erwartungen, die für beide Länder gleich sind, kann es aber auch zu länderspezifischen Unterschieden kommen, die vorwiegend institutionell begründet werden können, sich aber auf die individuellen Ursachen auswirken. Obwohl es sich bei beiden Ländern um meritokratische Gesellschaften handelt, in denen das Leistungsprinzip vorherrscht, steht in der Schweiz das Individuum am Arbeitsmarkt stärker im Zentrum der Aufmerksamkeit, während in Deutschland, geprägt durch die konservative, wohlfahrtsstaatliche Tradition, die Rolle der Familie stärker betont wird. Vor diesem Hintergrund ist zu erwarten, dass in der Schweiz die Einkommen stärker von der individuellen Leistung, der Bildung, abhängen als in Deutschland (Hypothese 5), aber umgekehrt in Deutschland die sozialen Herkunftseffekte die Lohnhöhe stärker beeinflussen (Hypothese 6).

Um diese Zusammenhänge zu analysieren, wird in einem ersten Schritt untersucht, inwieweit die soziale Herkunft Einfluss auf die Bildung hat. In einem zweiten Schritt wird dann der Einfluss der sozialen Herkunft auf die Einkommenshöhe untersucht. Da die abhängige Variable der ersten Modellspezifikation, der höchste formale Bildungsabschluss, als kategoriales Merkmal vorliegt, wird ein Ordered Logit Modell geschätzt. Um anschließend zu untersuchen, wie sich der Einfluss der sozialen Herkunft und Bildung auf Lohnunterschiede gestaltet, werden 
in einem zweiten Analyseschritt lineare Regressionen geschätzt (vgl. zum methodischen Vorgehen z.B. Greene 2003).

Da insbesondere die Unterschiede zwischen beiden Ländern im Mittelpunkt des Interesses stehen, werden Interaktionsterme zwischen dem Land und den einzelnen unabhängigen Variablen in gepoolte Modelle aufgenommen. Bei Interaktionen zwischen z.B. einem kategorialen und einem metrischen Merkmal sieht die Regressionsgleichung mit zwei unabhängigen Variablen folgendermaßen aus:

(1) $Y_{i}^{*}=\beta_{0}+\beta_{1} X+\beta_{2} Z+\beta_{3} X Z+\varepsilon$

Die Parameter $\beta_{1}$ und $\beta_{2}$ repräsentieren die Haupteffekte zweier Variablen, $\beta_{3}$ den Interaktionseffekt dieser Merkmale. Bei Interaktionen zwischen einem kategorialen und einem metrischen Merkmal, z.B. der Geburtskohorte $(X)$ und dem Geschlecht (Z, mit 0 für Frauen und 1 für Männer), kann die Regressionsgleichung so formuliert werden, dass für Frauen $(Z=0)$ gilt: $\ln Y=\beta_{0}+\beta_{1} X+\varepsilon$ und für Männer $(Z=1)$ : $\ln Y=\beta_{0}+\beta_{1} X+\beta_{2}+\beta_{3} X$ bzw. $\ln Y=\beta_{0}+$ $\beta_{2}+\left(\beta_{1}+\beta_{3}\right) X$. Der Effekt des Geburtsjahrgangs auf Bildung ist damit für Frauen gleich $\beta_{1} X$ und für Männer gleich $\left(\beta_{1} X+\beta_{3} X\right)$. Ist $\beta_{3} X$ signifikant von Null verschieden, dann liegt eine Interaktion zwischen der Geburtskohorte und Geschlecht vor, d.h. der Einfluss unterscheidet sich für Männer und Frauen. Um zu prüfen, ob auch der Haupteffekt für Männer $\left(\beta_{1} X+\beta_{3} X\right)$ signifikant von Null verschieden ist, wird das statistische Verfahren des Post Hoc Probing angewendet (vgl. dazu ausführlich Aiken / West 1991: 14ff). Dazu werden die Standardfehler folgendermaßen berechnet:

(2) $S b=\sqrt{S_{11}+2(Z) S_{13}+(Z)^{2} S_{33}}$,

wobei $\mathrm{s}_{11}$ und $\mathrm{s}_{33}$ die Varianz von $\beta_{1}$ bzw. $\beta_{3}$ ist und $\mathrm{s}_{13}$ der Kovarianz zwischen $\beta_{1}$ und $\beta_{3}$ entspricht.

\section{Daten und Operationalisierung}

Um den Zusammenhang zwischen sozialer Herkunft, Bildung und Einkommen zu untersuchen, wird für Deutschland auf das Sozio-oekonomische Panel (SOEP) und das Schweizer HaushaltPanel (SHP) ${ }^{8}$ zurückgegriffen. Die beiden Datensätze sind für die folgenden Analysen besonders geeignet, da sie umfassende Informationen zum Humankapital und zur sozialen Herkunft beinhalten. Das SOEP wird seit 1984 jährlich erhoben (SOEP Group 2001). Seine thematischen Schwerpunkte sind Erwerbs- und Bildungsbiografien, Einkommen, Familienbildungsprozesse, Wohnen, Zeitverwendung, subjektive Indikatoren wie Zufriedenheiten oder Sorgen sowie ausführliche Informationen zur sozialen Herkunft. Alle Mitglieder eines Haushaltes, die mindestens 17 Jahre alt sind, werden jährlich mit einem Personenfragebogen befragt. Zusätzlich wird pro Haushalt von einem Mitglied ein Haushaltsfragebogen ausgefüllt, der z.B. Fragen zur Wohnsituation enthält. Da das SOEP eine Paneluntersuchung ist, werden dieselben Personen im jährlichen Rhythmus wiederbefragt, auch dann, wenn sie einen Haushalt verlassen haben.

Für die schweizerischen Analysen wird das Schweizer Haushalt-Panel (SHP) verwendet, das seit 1999 erhoben wird (vgl. Zimmermann et al. 2003). Die Umfrage Leben in der Schweiz deckt ebenso wie das SOEP eine breite Palette sozialwissenschaftlicher Fragestel-

8 Mit der Datennutzung ist folgende Erklärung abzugeben: Für die Publikation wurden die Daten „Leben in der Schweiz" verwendet, die vom Schweizer Haushalt-Panel (SHP) erhoben werden. Das SHP ist ein Projekt der Schweizer Stiftung für die Forschung in den Sozialwissenschaften FORS an der Universität Lausanne und wird vom Schweizerischen Nationalfonds zur Förderung der wissenschaftlichen Forschung finanziert. 
lungen ab. Alle Mitglieder eines Haushalts werden mit einem individuellen Fragebogen erfasst, zusätzlich wird pro Haushalt von einer Person ein Haushaltsfragebogen ausgefüllt. Auch hier liegen Informationen zur sozialen Herkunft vor. Aber während im SOEP Personen erst ab 17 Jahren befragt werden, liegt das Erstbefragungsalter in der Schweiz bereits bei 14 Jahren. Die Interviews werden mit CATI (Computer-Assisted Telephone Interviewing) erhoben, im SOEP durch persönliche Face-to-Face-Interviews mit „Paper and Pencil“ (PAPI) oder CAPI (Computer-Assisted Personal Interviewing). Die jeweilige Untersuchungseinheit ist in beiden Ländern bzw. Datensätzen die ständige Wohnbevölkerung in Privathaushalten.

In einem ersten Analyseschritt wird der Zusammenhang zwischen der sozialen Herkunft und der individuellen Bildung untersucht. Da die abhängige Variable, das höchste Bildungsniveau nach CASMIN, ${ }^{9}$ neben schulischen auch berufliche Bildungsabschlüsse umfasst und in Deutschland und der Schweiz universitäre Abschlüsse im internationalen Vergleich erst spät erreicht werden, werden als Untersuchungseinheit nur Personen bis zum Geburtsjahrgang 1973 ausgewählt und jüngere Geburtskohorten ausgeschlossen. Durch diese Abgrenzung soll gewährleistet werden, dass die Befragten das Bildungssystem abschließend durchlaufen haben. ${ }^{10}$ Außerdem beschränkt sich die Stichprobe nur auf Befragte, die im deutschen bzw. schweizerischen Bildungssystem auch ihren Abschluss erworben haben, denn das Erreichen eines bestimmten Bildungsniveaus hängt maßgeblich von der länderspezifischen Gestaltung des Systems ab. Die CASMIN-Klassifikation berücksichtigt neben den schulischen auch die beruflichen Bildungsabschlüsse, unterstellt eine aufsteigende Rangfolge der Abschlüsse und gewährleistet zusätzlich die internationale Vergleichbarkeit. Im SOEP ist diese Variable vorhanden, für die Schweiz wurden die vorhandenen Bildungsinformationen in CASMIN-Kategorien überführt. Tabelle 1 gibt einen Überblick zu den einzelnen Kategorien.

\section{Tabelle 1: CASMIN-Bildungsklassifikation}

\begin{tabular}{|c|c|}
\hline & Beschreibung \\
\hline $1 \mathrm{a}$ & $\begin{array}{l}\text { Inadequately completed general education- } \\
\text { ohne Abschluss }\end{array}$ \\
\hline $1 \mathrm{~b}$ & $\begin{array}{l}\text { General elementary education - } \\
\text { Haupt- / Volksschulabschluss }\end{array}$ \\
\hline $1 \mathrm{c}$ & $\begin{array}{l}\text { Basic vocational qualification / General elementary education and vocational qualification } \\
- \\
\text { Haupt- / Volksschulabschluss mit Abschluss einer Lehr- / Anlernausbildung oder Meister- / } \\
\text { Technikerausbildung }\end{array}$ \\
\hline $2 \mathbf{a}$ & $\begin{array}{l}\text { Intermediate vocational qualification / Intermediate general qualification and vocational } \\
\text { qualification - } \\
\text { Realschulabschluss (Mittlere Reife) mit Abschluss einer Lehr- /Anlernausbildung oder Meis- } \\
\text { ter- / Technikerausbildung }\end{array}$ \\
\hline $2 \mathrm{~b}$ & $\begin{array}{l}\text { Intermediate general qualification - } \\
\text { Realschulabschluss (Mittlere Reife) }\end{array}$ \\
\hline 2c_gen & $\begin{array}{l}\text { General maturity certificate- } \\
\text { Fachhochschulreife, Hochschulreife (Abitur bzw. Matura) }\end{array}$ \\
\hline 2c_voc & $\begin{array}{l}\text { Vocational maturity certificate / General maturity certificate and vocational qualification- } \\
\text { Fachhochschulreife, Hochschulreife (Abitur / Matura) mit Abschluss einer Lehr- / Anlern- } \\
\text { ausbildung oder Meister- / Technikerausbildung }\end{array}$ \\
\hline $3 \mathbf{a}$ & $\begin{array}{l}\text { Lower tertiary education - } \\
\text { Fachhochschulabschluss, Ingenieurschulabschluss }\end{array}$ \\
\hline
\end{tabular}

9 Vgl. zum Originalkonzept König et al. (1988), zur Weiterentwicklung Brauns / Steinmann (1999).

10 Obwohl es natürlich theoretisch möglich ist, dass auch noch in späteren Jahren Bildungsabschlüsse nachgeholt werden, kann durch diese Abgrenzung zumindest für den Großteil der Befragten angenommen werden, dass sie das höchste Bildungsniveau abschließend erreicht haben. 


\begin{tabular}{ll}
\hline & Beschreibung \\
\hline $\mathbf{3 b}$ & $\begin{array}{l}\text { Higher tertiary education - } \\
\text { Hochschulabschluss }\end{array}$ \\
\hline
\end{tabular}

Quelle: Brauns/ Steinmann (1999: 43).

Die neun Ursprungskategorien wurden zum einen aus Gründen der Übersichtlichkeit, zum anderen aus datentechnischen Gründen zu vier Kategorien zusammengefasst, da es für die Schweiz nicht möglich ist, auf Basis der im SHP erhobenen Bildungsvariable zwischen den Kategorien $1 \mathrm{c}$ bis $2 \mathrm{~b}$ zu differenzieren. (Noch) kein formaler Schulabschluss ( $1 \mathrm{a}$ ) bzw. eine geringe formale Bildung $(1 \mathrm{~b})$ wie sie durch den Abschluss der Pflichtschule angezeigt wird, werden zu CASMIN 0 (1ab) zusammengefasst. Personen, die den Hauptschulabschluss und zusätzlich eine berufliche Ausbildung abgeschlossen haben, sowie Personen mit mittlerer Reife (ohne und mit beruflichem Abschluss) werden unter CASMIN 1 (1c-2 b) subsumiert. In der Schweiz werden Personen, die eine abgeschlossene Lehre (aber keine Matura) haben, in diese Kategorie aufgenommen. CASMIN 2 (2c gen / 2c voc) umfasst Personen, die das Abitur als höchsten schulischen Bildungsabschluss besitzen und teilweise zusätzlich noch eine berufliche Ausbildung absolviert haben. Schließlich werden Personen mit einem tertiären Abschluss Fachhochschule oder Universität - zu CASMIN 3 (3ab) umcodiert. ${ }^{11}$

Da im Zuge der Bildungsexpansion jüngere Jahrgänge zunehmend höhere Abschlüsse erreichen, wird als erklärendes Merkmal in die Untersuchungen die Geburtskohorte aufgenommen. Damit die Effekte besser interpretierbar sind, wird vom Geburtsjahr der Wert 1900 subtrahiert. Größere Werte zeigen jüngere Kohorten an. Der Wertebereich reicht empirisch in Deutschland von 3 (entspricht dem Geburtsjahrgang 1903) bis 73 (1973). Für die Schweiz liegen die Werte zwischen 4 und 73. Auch das Geschlecht wird als soziodemografisches Merkmal in die Analysen aufgenommen ebenso die Nationalität. Dabei wird nach Personen unterschieden, die die inländische Staatsbürgerschaft, d.h. einen deutschen bzw. schweizerischen Pass, oder eine andere Staatsbürgerschaft besitzen.

Während in der klassischen Studie zur Statuszuweisung von Blau und Duncan (1967) sowie auch in neueren Untersuchungen zu Einkommensunterschieden (z.B. Hirschel 2004) nur die sozioökonomische Situation des Vaters berücksichtigt wird, erscheint es sinnvoll, die Merkmale beider Elternteile in den folgenden Analysen zu betrachten. Denn welcher Einfluss wichtiger ist, ist wissenschaftlich umstritten (vgl. Fußnote 7). Aus diesem Grund wird hier als soziales Herkunftsmerkmal der höchste formale Bildungsabschluss des Elternhauses, d.h. entweder derjenige des Vaters oder derjenige der Mutter aufgenommen. Diese Informationen werden ebenfalls mittels der CASMIN-Klassifikation abgebildet. Dazu wurden sowohl im SOEP als auch im SHP die Informationen zur Bildung der Eltern aus den Datensätzen zur sozialen Herkunft in CASMIN überführt und entsprechend recodiert. In die Schätzungen wird dann die jeweils höhere Bildungsinformation aufgenommen. Da für ca. $14 \%$ der Befragten keine Informationen zum Bildungsabschluss der Eltern vorliegen, wird in die Modellschätzungen zusätzlich eine Dummy-Variable aufgenommen, die die Information enthält, ob die Angabe vorhanden ist oder nicht. Da keine Informationen zur finanziellen Situation des Elternhauses in der Kindheit vorliegen, wird das (Berufs-)Prestige der Eltern als Näherungsvariable für die soziale Lage verwendet. Diese Informationen wurden retrospektiv erhoben. Es wird die Klassifikation nach Treiman (1977), die so genannte „Standard International Occupational Prestige Scale“ (SIOPS), verwendet. Die Wahl dieser Skala kann einerseits datentechnisch begründet werden, da sie für Deutschland und die Schweiz vorhanden ist. Anderer-

11 In Deutschland liegen für 222 Befragte $(1,7 \%)$ keine Angaben zur Bildung vor. In der Schweiz sind diese Informationen für alle Befragten vorhanden, entweder auf Basis eigener Angaben oder durch Proxy-Informationen. 
seits bietet die Treiman-Skala den Vorteil, dass die Prestigewerte auf Basis von Bevölkerungsumfragen in fast 60 Ländern ermittelt wurden und sie somit für internationale Vergleiche geeignet ist. Auch hier wird wieder der höchste Wert des Elternhauses verwendet. Da der Anteil der fehlenden Werte wiederum relativ groß ist, wird zusätzlich eine Variable aufgenommen, die Auskunft darüber gibt, ob ein gültiger Wert vorliegt oder nicht. ${ }^{12}$

Um dann in einem zweiten Schritt den Zusammenhang zwischen der sozialen Herkunft, der Bildung und dem Einkommen zu untersuchen, werden jeweils alle Vollzeit und Teilzeit erwerbstätigen, abhängigen Beschäftigten ab 17 Jahren, die ein Einkommen von größer als Null haben, berücksichtigt. ${ }^{13}$ Die Erwerbseinkommen werden im SOEP sowohl auf Monats- als auch auf Jahresbasis erfasst. In den folgenden Untersuchungen wird das jährliche Bruttoerwerbseinkommen ${ }^{14}$ aus den so genannten Equivalent-Files verwendet, weil auch im SHP jahresbezogene Werte vorliegen und bei dieser Variable fehlende Werte imputiert worden sind. Um für Erwerbsumfangs- und Selektionseffekte zu kontrollieren, werden Stundenlöhne berechnet. Dazu wird auf Basis der Formel von Szydlik (1993) der Stundenlohn wie folgt berechnet: ${ }^{15}$

(3) $y=\ln \left(\frac{\text { labinc } / 30.4375}{\text { whours } / 7}\right)$

wobei labinc, dem monatlichen Erwerbseinkommen (brutto) entspricht und whours den verträglich festgelegten Arbeitsstunden.

Auf Basis der im 2. Abschnitt dargestellten und diskutierten Ansätze lassen sich verschiedene Einflussfaktoren für die Erklärung der Erwerbseinkommen operationalisieren. Als soziodemografische Hintergrundmerkmale werden das Geschlecht, das Alter (linear und quadriert) sowie die Nationalität aufgenommen. Der Einfluss der sozialen Herkunft wird wiederum über das höchste Bildungsniveau des Elternhauses nach der CASMIN-Klassifikation berücksichtigt. Um die humankapitaltheoretischen Überlegungen zu untersuchen, wird der höchste formale Bildungsabschluss des Befragten in die Modellschätzungen aufgenommen. Neben der formalen Bildung werden weitere, nach der Humankapitaltheorie relevante Variablen getestet. Das sind für Deutschland und die Schweiz (1) die Berufserfahrung ${ }^{16}$ und (2) das Merkmal, ob jemand in den letzten Jahren einen Weiterbildungskurs besucht hat. Für beide Länder wird die Berufserfahrung aus den Biographieangaben zur Berufstätigkeit auf Basis von Spells generiert. Diese Angaben liegen in beiden Datensätzen auf Jahresbasis vor. Durch das Aufsummieren der einzelnen Anfangs- und Endzeitpunkte der jeweiligen Erwerbstätigkeitsspells kann die Dauer seit Eintritt in den Arbeitsmarkt unter Berücksichtigung von Phasen der Inaktivität operationalisiert werden. Dieses Vorgehen hat den Vorteil, dass dadurch zeitweilige Phasen der Arbeitslosigkeit und beruflicher Inaktivität abgebildet werden können, die zu Humankapital-

12 Ca. ein Drittel der Befragten können keine Angaben zum Berufsprestige der Eltern machen.

13 Da sich unter 17-Jährige zumeist noch in der Ausbildung befinden und Personen im (Aus-)Bildungssystem, ebenso wie Wehr- und Zivildienstleistende in den folgenden Analysen als Untersuchungspopulation ausgeschlossen werden, da sie in der Regel kein Erwerbseinkommen haben, ist die Altersbegrenzung gerechtfertigt. Auch Selbständige werden ebenso wie Frauen, die sich im Mutterschaftsurlaub befinden, aus den Analysen ausgeschlossen.

14 Die Einkommensangaben werden brutto berücksichtigt, weil dadurch steuerliche Einflüsse, die sich aus dem Haushaltszusammenhang ergeben, ausgeklammert werden. Außerdem werden die Erwerbseinkommen in den multivariaten Analysen logarithmiert.

15 Alternativ wurde zur Konsistenzprüfung der Stundenlohn auch nach der Formel von Nyhus / Pons (2005) berechnet, die explizit Bruttojahreseinkommen verwenden. Die Ergebnisse weisen jedoch keine nennenswerten Unterschiede auf.

16 Die Berufserfahrung wird in dieser Modellspezifikation anstelle des Alters aufgenommen, da beide miteinander korrelieren und es dadurch zu Verzerrungen kommen kann. 
entwertungen und daraus folgend zu Einkommensverlusten führen können. Die Berufserfahrung wird linear und quadriert aufgenommen. Der Weiterbildungsindikator wird als Dummy aufgenommen und bildet ab, ob jemand in den letzten drei Jahren einen Fortbildungskurs besucht hat oder nicht.

\section{Empirische Befunde}

\subsection{Der Einfluss der sozialen Herkunft auf Bildung}

In diesem Abschnitt wird zunächst untersucht, inwieweit beim Bildungserwerb in Deutschland und der Schweiz soziale Herkunftseffekte zum Tragen kommen. Um valide Aussagen darüber treffen zu können, ob die Chancengleichheit in einem der beiden Länder größer ist, wird im Folgenden ein Ordered Logit Modell auf Basis eines gepoolten Datensatzes geschätzt, das Interaktionseffekte der erklärenden Merkmale mit dem Land enthält (Modell 1). In die Modellspezifikation wird die Herkunft zum einen über den höchsten Bildungsabschluss des Elternhauses, zum anderen über das Berufprestige, welches eine Näherungsvariable für die soziale Lage in der Kindheit darstellt, abgebildet. ${ }^{17}$ Tabelle 2 gibt einen Überblick über die empirischen Ergebnisse.

Bei Betrachtung der soziodemografischen Merkmale zeigt sich, dass Frauen sowohl in Deutschland als auch der Schweiz immer noch geringere Bildungsabschlüsse aufweisen als Männer. Allerdings werden die geschlechtsspezifischen Unterschiede für jüngere Kohorten im Zuge der Bildungsexpansion deutlich geringer wie der negative Interaktionsterm zwischen dem Geschlecht und der Geburtskohorte anzeigt. Insgesamt sind aber die Unterschiede der Geschlechter im Bildungsniveau in Deutschland schwächer ausgeprägt als in der Schweiz. Das bestätigt auch frühere Befunde z.B. der OECD (2004: 84), wonach in der Schweiz neben Japan und der Türkei Männer immer noch höhere Abschlüsse erreichen als Frauen.

Tabelle 2: Determinanten des Bildungserfolgs (Ordered Logit Modell)

\begin{tabular}{|c|c|c|}
\hline & \multicolumn{2}{|c|}{ Modell 1} \\
\hline & Koeff. & z-Wert \\
\hline Land & -.07 & $(-0.36)$ \\
\hline \multicolumn{3}{|l|}{ Schweiz } \\
\hline \multicolumn{3}{|l|}{ Deutschland } \\
\hline Geschlecht & $2.77 * *$ & $(23.53)$ \\
\hline \multicolumn{3}{|l|}{ Frauen } \\
\hline \multicolumn{3}{|l|}{ Männer } \\
\hline Geburtskohorte & $.04 * *$ & $(18.50)$ \\
\hline \multicolumn{3}{|l|}{ Linear } \\
\hline Nationalität & $-.56 * *$ & $(-2.61)$ \\
\hline \multicolumn{3}{|l|}{ Inländer } \\
\hline \multicolumn{3}{|l|}{ Ausländer } \\
\hline Bildung Eltern & $-.83 * *$ & $(-9.54)$ \\
\hline CASMIN 1 & $.53 * *$ & $(6.76)$ \\
\hline CASMIN 0 & $1.04 * *$ & (9.09) \\
\hline CASMIN 2 & $-.66 * *$ & $(-4.40)$ \\
\hline
\end{tabular}

CASMIN 3

Angabe fehlt

17 Alternativ wurden auch Modelle geschätzt, in die jeweils nur eines der beiden Merkmale aufgenommen wurde, da das Berufsprestige nicht unabhängig von der Bildung ist. Dabei zeigt sich, dass die Ergebnisse keine nennenswerten Unterschiede zu der dargestellten Spezifikation aufweisen und vor diesem Hintergrund auf eine Darstellung verzichtet werden kann. 


\begin{tabular}{lrl}
\hline & Modell 1 \\
\hline & Koeff. & z-Wert \\
\hline Prestige Eltern & $.03^{* *}$ & $(8.13)$ \\
linear & $.99^{* *}$ & $(5.30)$ \\
Angabe fehlt & & \\
Interaktionseffekte & $-.04^{* *}$ & $(-18.06)$ \\
Geschlecht*Geburtskohorte & $-.33^{* *}$ & $(-5.12)$ \\
Geschlecht*Land & $-.01^{*}$ & $(-2.45)$ \\
Geburtskohorte*Land & $-.73^{* *}$ & $(-2.91)$ \\
Nationalität*Land & .03 & $(0.30)$ \\
Bildung*Land & $.58^{* *}$ & $(5.15)$ \\
CASMIN1*Land & $.27^{*}$ & $(2.11)$ \\
CASMIN0*Land & .08 & $(0.46)$ \\
CASMIN2*Land & & \\
CASMIN3*Land & $.01^{* *}$ & $(3.03)$ \\
Angabe fehlt*Land & .26 & $(1.26)$ \\
Prestige*Land & & \\
linear*Land & 1.12 & \\
Angabe fehlt*Land & 4.47 & \\
\hline Cut-Point 1 & 5.05 & \\
Cut-Point 2 & 21.871 \\
Cut-Point 3 & .11 \\
\hline n & & \\
\hline Pseudo-R ${ }^{2}$ & & \\
\hline
\end{tabular}

Anmerkungen: Referenzkategorien kursiv.

Signifikanzniveaus: $* * \mathrm{p}<.01, * \mathrm{p}<.05,{ }^{+} \mathrm{p}<.10$.

z-Werte in Klammern.

Quellen: SOEP 2003 und SHP 2003 (gepoolt), eigene Berechnungen.

Auch bei der Nationalität zeigen sich länderspezifische Unterschiede. Zwar sind in beiden Ländern ausländische Staatsbürger beim Bildungserwerb im Nachteil, aber in Deutschland ist die Wahrscheinlichkeit für nicht-deutsche Bürger wesentlich schlechter im deutschen Bildungssystem ein höheres Bildungsniveau zu erreichen als für Nicht-Schweizer im schweizerischen System, wie der auf dem 1-\%-Niveau signifikante, negative Interaktionseffekt anzeigt.

Um die Interaktionseffekte für die soziale Herkunft einfacher interpretieren und besser direkt vergleichen zu können, werden in Tabelle 3 die Ergebnisse für den Einfluss der sozialen Herkunft (Bildung und Berufsprestige des Elternhauses) anschaulicher dargestellt. ${ }^{18}$ Werden die herkunftsspezifischen Unterschiede auf den Bildungserwerb betrachtet, dann zeigt sich für beide Länder, dass das individuelle Bildungsniveau maßgeblich durch die soziale Herkunft beeinflusst wird. Je bildungsferner das Elternhaus ist, desto geringer sind die Chancen auf einen höheren Bildungsabschluss. Aber auch das Berufsprestige der Eltern hat in beiden Ländern einen positiven Einfluss auf den Bildungserfolg der Kinder. Somit kann auf Basis der empirischen Befunde Hypothese 1, wonach in Deutschland und der Schweiz der individuelle Bildungserfolg deutlich von der sozialen Herkunft abhängt, bestätigt werden.

18 Die Haupteffekte für Deutschland ergeben sich, indem die jeweiligen Koeffizienten wie z.B. das Geschlecht und der Interaktionsterm (Geschlecht*Land) aufsummiert werden. 
Tabelle 3: Der Zusammenhang von sozialer Herkunft und Bildung im Ländervergleich

\begin{tabular}{lrl}
\hline & Modell 1 \\
\hline & Koeff. & z-Wert \\
\hline Bildung Vater*Land & $-.83^{* *}$ & $(-9.54)$ \\
CASMIN 0 & .03 & $(0.30)$ \\
Schweiz & $-.80^{* *}$ & $(-17.25)$ \\
Interaktionseffekt & $.54^{* *}$ & $(6.76)$ \\
Deutschland & $.58^{* *}$ & $(5.15)$ \\
CASMIN 2 & $1.12^{* *}$ & $(13.77)$ \\
Schweiz & $1.04^{* *}$ & $(9.09)$ \\
Interaktionseffekt & $.27^{* *}$ & $(2.11)$ \\
Deutschland & $1.31^{* *}$ & $(23.99)$ \\
CASMIN 3 & $-.66^{* *}$ & $(-4.40)$ \\
Schweiz & .08 & $(0.46)$ \\
Interaktionseffekt & $-.58^{* *}$ & $(-8.88)$ \\
Deutschland & & \\
Angabe Bildung fehlt & & \\
Schweiz & & \\
Interaktionseffekt & & \\
Deutschland & & \\
Prestige Vater*Land & $.03^{* *}$ & $(8.13)$ \\
linear & $.01^{* *}$ & $(3.03)$ \\
Schweiz & $.04^{* *}$ & $(19.82)$ \\
Interaktionseffekt & $.99^{* *}$ & $(5.30)$ \\
Deutschland & .26 & $(1.26)$ \\
Angabe Prestige fehlt & $1.25^{* *}$ & $(-1.26)$ \\
Schweiz & & \\
Interaktionseffekt & & \\
Deutschland & &
\end{tabular}

Anmerkungen: Die Signifikanzen für die Geschlechtseffekte der Männer wurden auf Basis des statistischen Post Hoc Probing berechnet (vgl. Abschnitt 3). Referenzkategorien kursiv.

Signifikanzniveaus: $* * \mathrm{p}<.01,{ }^{*} \mathrm{p}<.05,{ }^{+} \mathrm{p}<.10$. Standardfehler in Klammern.

Quellen: SOEP 2003 und SHP 2003, eigene Berechnungen auf Basis Tabelle 2.

Allerdings ist vor dem Hintergrund der Pisa-Befunde zu erwarten, dass der Einfluss des Elternhauses in Deutschland noch stärker ist als in der Schweiz (Hypothese 2). In Einklang mit dieser Annahme zeigen die empirischen Befunde auch die erwarteten länderspezifischen Unterschiede in der Stärke des Zusammenhangs. In Deutschland sind die herkunftsbedingten Bildungseffekte wesentlich stärker als in der Schweiz. Wenn mindestens ein Elternteil Abitur oder sogar einen Hochschulabschluss hat, dann sind die Chancen auf höhere Bildung signifikant größer als in der Schweiz. Gleiches gilt auch für das Berufsprestige. Dieses Ergebnis untermauert die aktuellen PISA-Befunde, wonach die Varianzaufklärung zwischen der sozialen Herkunft und der mathematischen Kompetenz in Deutschland mit $22.8 \%$ höher ist als in der Schweiz mit 16.8 \% (vgl. Prenzel et al. 2004: 249).

\subsection{Der Zusammenhang zwischen sozialer Herkunft, Bildung und Erwerbseinkommen}

Nachdem gezeigt werden konnte, dass insbesondere in Deutschland aber auch der Schweiz die soziale Herkunft eine zentrale Rolle beim Bildungserwerb einnimmt, soll nun untersucht werden, welchen Einfluss die soziale Herkunft und die Bildung auf den individuellen Arbeitsmarkterfolg haben. Um diesen Zusammenhang zu untersuchen, wird im Folgenden in zwei Analyseschritten vorgegangen. Zunächst wird ein Modell, das soziodemografische Hinter- 
grundmerkmale ${ }^{19}$ sowie eine Information zur sozialen Herkunft enthält, hier höchster Bildungsabschluss des Elternhauses, geschätzt (Modell 2). ${ }^{20}$ Anschließend wird zusätzlich die Bildungsinformation der Befragten aufgenommen (Modell 3 a) und in einem weiteren Modell (3 b) noch für spezifische Humankapitalindikatoren kontrolliert.

Wird der Zusammenhang zwischen dem Bildungsniveau des Elternhauses und der Lohnhöhe ohne Berücksichtigung der (eigenen) Bildung des Befragten betrachtet, dann zeigt sich, dass die soziale Herkunft in beiden Ländern einen signifikanten Einfluss auf die Erwerbseinkommen hat (vgl. Tabelle 4, Modell 2). Je höher das Bildungsniveau des Elternhauses ist, desto höher sind die Erwerbseinkommen der Kinder. Hat mindestens ein Elternteil als höchsten Abschluss nur die Pflichtschule erreicht, dann sind die Einkommen signifikant geringer als bei Personen, bei denen mindestens ein Elternteil eine mittlere Bildung hat. Wenn dagegen Abitur bzw. Matura oder zusätzlich ein Hochschulabschluss vorliegen, dann steigen die Einkommen signifikant. Allerdings zeigen sich hier im Gegensatz zur Chancengleichheit keine länderspezifischen Unterschiede wie die nicht signifikanten Interaktionsterme zwischen dem Land und der Bildungsvariable der Eltern anzeigen. ${ }^{21}$ Doch wie sieht der Zusammenhang zwischen der sozialen Herkunft und der individuellen Lohnhöhe aus, wenn für die eigene Bildung (Modell 3) bzw. zusätzlich spezifisches Humankapital (Modelle 4) kontrolliert wird?

Wenn für die (eigene) Bildung kontrolliert wird, dann verliert die Bildung des Elternhauses sowohl in Deutschland als auch der Schweiz an Bedeutung (vgl. Tabelle 4, Modell 3). Lediglich wenn die Eltern nur das niedrigste Bildungsniveau als höchsten Abschluss vorweisen können, gibt es einen negativen Effekt auf die Lohnhöhe, der unabhängig vom Einkommen wirkt. Dieser Zusammenhang ist in Deutschland ausgeprägter als in der Schweiz, wie der auf dem $10 \%$ Niveau signifikante Interaktionsterm anzeigt. Insofern kann Hypothese 6 unter Vorbehalt bestätigt werden. Insgesamt ist der direkte Zusammenhang zwischen der sozialen Herkunft und der Lohnhöhe aber für beide Länder schwächer als erwartet. Es scheint, als ob der Einfluss der Herkunft beim Bildungserwerb zwar die Chancengleichheit negativ beeinflusst, insgesamt verliert der Einfluss der sozialen Herkunft auf die Erwerbseinkommenshöhe aber an Bedeutung, wenn für das individuelle Bildungsniveau kontrolliert wird. Insofern kann Hypothese 4, wonach auch ein direkter Effekt der sozialen Herkunft auf Lohnunterscheide vorliegt, auf Basis der hier durchgeführten Analysen für beide Länder nur bedingt bestätigt werden. Aber Hypothese 3, wonach die individuelle Bildung das zentrale Merkmal für den individuellen Arbeitsmarkterfolg ist, kann bestätigt werden. Je höher die formale Bildungsqualifikation, desto höher sind die Erwerbseinkommen. Das gilt für beide Länder gleichermaßen und es zeigen sich auch keine signifikanten Unterschiede in der Bedeutung des Bildungseffektes für

19 Da in beiden Modellen sowohl die Anzahl als auch die Kodierung der Merkmale identisch sein muss, kann kein Merkmal mit in die Spezifikationen aufgenommen werden, das für Deutschland zwischen Ost und West differenziert.

20 Das Berufsprestige wurde als Näherungsvariable für die soziale Lage in die Modellschätzungen zur Chancengleichheit aufgenommen, denn es zeigt sich, dass das Einkommen des Elternhauses den Bildungserfolg in der Kindheit beeinflusst (Mulligan 1999; Dustmann 2004). Da Bildung und Berufsprestige der Eltern aber miteinander zusammenhängen, wird an dieser Stelle darauf verzichtet, das Prestige aufzunehmen, da für die Erwerbseinkommen nicht die Lage in der Kindheit, sondern die unterschiedlichen Sozialisationsbedingungen in bildungsfernen und -nahen Elternhäusern wichtiger sind. Das sind nach Boudon (1974: 29ff) die primären Effekte, bei denen es sich um den langfristigen Einfluss der Leistungsentwicklung handelt, die durch die elterliche Förderung im Sozialisationsprozess zustande kommt.

21 Wenn die Modelle jedoch getrennt für Männer und Frauen geschätzt werden, dann zeigt sich, dass in Deutschland Töchter aus Akademikerhaushalten deutlich stärker beim Einkommenserwerb von ihrer Herkunft profitieren als in der Schweiz. Für Männer zeigen sich dagegen keine signifikanten Unterschiede. 
Deutschland und die Schweiz. Hypothese 5 kann somit für den Einfluss der formalen Bildungsqualifikation nicht bestätigt werden.

Tabelle 4: Zum Zusammenhang von Herkunft, Humankapital und Einkommen

\begin{tabular}{|c|c|c|c|c|c|c|}
\hline & Koeff. & z-Wert & Koeff. & z-Wert & Koeff. & z-Wert \\
\hline & \multicolumn{2}{|c|}{ Modell 2} & \multicolumn{2}{|c|}{ Modell 3} & \multicolumn{2}{|c|}{ Modell 4} \\
\hline \multicolumn{7}{|l|}{ Land } \\
\hline Deutschland & $-.52 * *$ & $(-2.82)$ & $-.65 * *$ & $(-3.69)$ & $-.20 * *$ & $(-3.25)$ \\
\hline \multirow{2}{*}{\multicolumn{7}{|c|}{ Geschlecht }} \\
\hline & & & & & & \\
\hline Männer & $.25^{* *}$ & $(11.12)$ & $.19^{* *}$ & $(9.03)$ & $.21^{* *}$ & $(8.34)$ \\
\hline \multicolumn{7}{|l|}{ Nationalität } \\
\hline Ausländer & $-.08 *$ & $(-2.34)$ & -.04 & $(-1.14)$ & -.01 & $(-0.20)$ \\
\hline \multicolumn{7}{|l|}{ Alter } \\
\hline Linear & $.09 * *$ & (11.63) & $.08^{* *}$ & $(10.03)$ & & \\
\hline quadriert & $-.00 * *$ & $(-10.31)$ & $-.00 * *$ & $(-8.59)$ & & \\
\hline \multicolumn{7}{|l|}{ Bildung Elternhaus } \\
\hline \multicolumn{7}{|l|}{ CASMIN 1} \\
\hline CASMIN 0 & $-.13 * *$ & $(-4.07)$ & $-.07 *$ & $(-2.33)$ & .05 & -1.36 \\
\hline CASMIN 2 & $.11 * *$ & (3.64) & .05 & $(1.58)$ & $.06^{+}$ & 1.68 \\
\hline CASMIN 3 & $.15^{* *}$ & $(4.14)$ & .02 & $(0.49)$ & .04 & 0.90 \\
\hline Angabe fehlt & -.06 & $(-1.06)$ & -.03 & $(-0.59)$ & $-.14^{+}$ & -1.95 \\
\hline \multicolumn{7}{|l|}{ Bildung Befragter } \\
\hline \multicolumn{7}{|l|}{ CASMIN 1} \\
\hline CASMIN 0 & & & $-.18 * *$ & $(-3.92)$ & $-.21 * *$ & $(-3.45)$ \\
\hline CASMIN 2 & & & $.21 * *$ & $(7.92)$ & $.20 * *$ & $(6.63)$ \\
\hline CASMIN 3 & & & $.36^{* *}$ & $(12.52)$ & $.40^{* *}$ & (11.84) \\
\hline \multicolumn{7}{|l|}{ Berufserfahrung } \\
\hline linear & & & & & $.04 * *$ & $(7.43)$ \\
\hline quadriert & & & & & $-.00 * *$ & $(-6.01)$ \\
\hline \multicolumn{7}{|l|}{ Kurse } \\
\hline \multicolumn{7}{|l|}{ nein } \\
\hline ja & & & & & $.11^{* *}$ & $(4.29)$ \\
\hline \multicolumn{7}{|l|}{ Interaktionseffekte } \\
\hline Geschlecht"Land & $.09 * *$ & $(3.74)$ & $.13 * *$ & $(5.46)$ & $.10^{* *}$ & $(3.66)$ \\
\hline Alter*Land (Berufserfah- & -.00 & $(-0.36)$ & .00 & $(0.70)$ & $-.01 * *$ & $(-2.76)$ \\
\hline rung*Land) & .00 & $(0.99)$ & -.00 & $(-0.25)$ & $.00^{* *}$ & $(2.66)$ \\
\hline \multicolumn{7}{|l|}{$\begin{array}{l}\text { Alter }{ }^{2 *} \text { Land (Berufserfah- } \\
\text { rung } 2 * \text { Land) }\end{array}$} \\
\hline Nationalität*Land & -.01 & $(-0.25)$ & -.01 & $(-0.35)$ & $-.09^{+}$ & $(-1.85)$ \\
\hline Kurse* Land & & & & & .03 & $(0.99)$ \\
\hline \multicolumn{7}{|l|}{ Bildung Eltern*Land } \\
\hline \multicolumn{7}{|l|}{ CASMIN $1 *$ Land } \\
\hline CASMIN0*Land & -.05 & $(-1.29)$ & -.06 & $(-1.73)$ & -.05 & $(-1.34)$ \\
\hline CASMIN $2 *$ Land & .02 & $(0.38)$ & -.03 & $(-0.64)$ & -.01 & $(-0.25)$ \\
\hline CASMIN3*Land & .06 & $(1.54)$ & .05 & $(1.12)$ & .04 & $(0.95)$ \\
\hline Angabe fehlt* Land & -.05 & $(-0.80)$ & -.04 & $(-0.61)$ & .05 & $(0.68)$ \\
\hline \multicolumn{7}{|l|}{ Bildung*Land } \\
\hline CASMIN 1 *Land & & & & & & \\
\hline CASMIN0*Land & & & -.01 & $(-0.14)$ & .10 & $(1.52)$ \\
\hline CASMIN2*Land & & & $-.11 * *$ & $(-3.34)$ & $-.07^{+}$ & $(-1.72)$ \\
\hline CASMIN3*Land & & & -.01 & $(-0.40)$ & -.02 & $(-0.50)$ \\
\hline Konstante & $.96 * *$ & (5.79) & $1.19^{* *}$ & $(7.50)$ & 2.51 & (43.28) \\
\hline
\end{tabular}




\begin{tabular}{|c|c|c|c|c|c|c|}
\hline & Koeff. & z-Wert & Koeff. & z-Wert & Koeff. & z-Wert \\
\hline & \multicolumn{2}{|c|}{ Modell 2} & \multicolumn{2}{|c|}{ Modell 3} & \multicolumn{2}{|c|}{ Modell 4} \\
\hline $\mathrm{n}$ & \multicolumn{2}{|c|}{9.080} & \multicolumn{2}{|c|}{8937} & \multicolumn{2}{|c|}{6553} \\
\hline $\mathrm{R}^{2}$ & \multicolumn{2}{|c|}{.32} & \multicolumn{2}{|c|}{.38} & & .37 \\
\hline
\end{tabular}

Anmerkungen: Referenzkategorien kursiv. Signifikanzniveaus: ${ }^{* *} \mathrm{p}<.01,{ }^{*} \mathrm{p}<.05,{ }^{+} \mathrm{p}<.10$. Standardfehler in Klammern.

Quellen: SOEP und SHP 2003, eigene Berechnungen.

Zuletzt werden neben der Bildung noch weitere, spezifische Humankapitalindikatoren in die Analyse aufgenommen (Modell 4): Das ist zum einen die Berufserfahrung, die ein besserer Indikator für Lerneffekte und Erfahrungswerte ist als das Lebensalter, da hierbei Erwerbsunterbrechungen aufgrund von Arbeitslosigkeit und insbesondere für Frauen durch Mutterschaftsurlaub entstehen können. ${ }^{22}$ Zum anderen gilt als relevant, ob die Befragten in den letzten Jahren einen beruflichen Weiterbildungskurs besucht haben. Dabei zeigt sich, dass Berufserfahrung und Weiterbildung ganz im Sinne der humankapitaltheoretischen Überlegungen die Lohnhöhe positiv beeinflussen (Tabelle 4, Modell 4). Doch während es in Deutschland und der Schweiz keinen Unterschied durch die Teilnahme an Weiterbildungskursen gibt, hat in der Schweiz die Berufserfahrung einen stärkeren Einfluss auf die Lohnhöhe als in Deutschland. Insofern kann zumindest für den Humankapitalindikator Berufserfahrung Hypothese 5 bestätigt werden, wonach Produktivitätsunterschiede auf Basis von Humankapital am schweizerischen Arbeitsmarkt wichtiger sind als am deutschen.

\section{Ausblick und sozialpolitische Implikationen}

Die ungleiche Verteilung von Einkommen im Allgemeinen und Erwerbseinkommen im Besonderen ist seit jeher ein viel diskutiertes Thema in Öffentlichkeit und Politik. So unterstreicht auch die Mediendebatte in Deutschland über die Herausbildung einer so genannten „,neuen Unterschicht“, teilweise auch als „Prekariat" bezeichnet, die bestehende Aktualität des Themas. Aber auch in der Schweiz sind Themen wie Chancengleichheit und Einkommensungleichheit in der öffentlichen Diskussion präsent. Doch wodurch kommen Unterschiede beim Bildungserwerb und im Einkommenserwerbsprozess zustande? Welche Rolle spielt bei allem die soziale Herkunft? Um diese Zusammenhänge aufzuzeigen, wurde im Rahmen dieser Studie der Frage nachgegangen, welche Rolle die soziale Herkunft im Statuszuweisungsprozess einnimmt, der einerseits vom individuellen Bildungserfolg und andererseits vom Arbeitsmarkterfolg abhängt.

Bei der Rechtfertigung von Lohnunterschieden wird in den modernen Gesellschaften primär auf individuelle Unterschiede in der Produktivität sowie in der Leistungsbereitschaft verwiesen. Die formale Bildung als Proxy für Produktivität ist somit ein zentrales Kriterium, um Leistungsunterschiede und dadurch Lohndifferenzen zu legitimieren. Damit dieser Rechtfertigungsmechanismus jedoch zur sinnvollen Argumentation herangezogen werden kann, müssen die Ausgangsbedingungen, die dafür sorgen, dass gewisse gesellschaftliche Positionen erreicht werden können, für alle Mitglieder einer Gesellschaft gleich sein. Doch die Realität sieht anders aus. Sowohl in Deutschland als auch der Schweiz ist der individuelle Bildungserfolg eng an die soziale Herkunft gekoppelt und Chancengleichheit besteht faktisch nur auf dem Papier. Denn die Bildungssysteme in Deutschland und der Schweiz sind derart organisiert und strukturiert, dass die soziale Herkunft, das Elternhaus, einen maßgeblichen Einfluss auf den Bildungserfolg hat. Damit gestaltet sich bereits die erste Hürde auf dem Weg zum Ar-

22 Da Alter und Berufserfahrung miteinander korrelieren, wird nur eines der beiden Merkmale in die jeweilige Modellspezifikation aufgenommen. 
beitsmarkterfolg, gemessen über die Lohnhöhe, derart, dass in Deutschland und der Schweiz einige Personen von vorneherein viel schlechtere Chancen haben als andere, eine gute Ausgangsposition in Form von hoher Bildung zu erreichen und somit auch die Einkommenserzielungschancen erschwert sind.

Doch auch wenn die Bildung ein zentraler Einflussfaktor für die Lohnhöhe ist, so kann das Humankapital nicht alle Unterschiede erklären. Neben dem Alter, dem Geschlecht und der Nationalität spielt insbesondere auch die soziale Herkunft eine Rolle und zwar zumindest derart, dass Kinder aus unteren Bildungsschichten wesentlich schlechtere Startbedingungen beim Bildungserwerb haben. Der erwartete direkte Zusammenhang zwischen der sozialen Herkunft und der Lohnhöhe ist in beiden Nationen zwar auf Basis der hier durchgeführten empirischen Analysen schwächer als erwartet, aber zumindest zeigt sich, dass Personen aus den untersten Bildungsschichten, wenn nur für die eigene Bildung kontrolliert wird, gegenüber Kindern aus höheren Bildungsschichten benachteiligt sind. Insgesamt scheint es so zu sein, dass der Einfluss der sozialen Herkunft auf die Lohnhöhe bereits beim Bildungserwerb zum Tragen kommt. Die Chancengleichheit wird negativ durch die Herkunft beeinflusst, hat aber jemand einmal den „Sprung“ zu höherer Bildung geschafft, dann sind die Erwerbseinkommen unabhängig(er) von der Herkunft.

Dennoch ist der Einfluss der Herkunft, die „Gnade der Geburt“, nicht zu unterschätzen und bereits durch die soziale Benachteiligung im Bildungssystem und auch am Arbeitsmarkt werden (systematisch) bestimmte Bevölkerungsgruppen ausgeschlossen, wodurch sich nicht nur das subjektive Wohlbefinden der Individuen verschlechtert, sondern auch andere individuelle Folgen wie soziale Isolation oder gesellschaftliche Folgen wie Kriminalität denkbar sind. Um dem entgegenzuwirken, muss darüber nachgedacht werden auf der Ebene der Bildungssysteme anzusetzen und die enge Verknüpfung zwischen Herkunft und Bildungsqualifikation neutralisiert werden. Denn die Höhe des verfügbaren Einkommens insgesamt hängt letztendlich von der Bildung ab oder wie Müller und Shavit (1998: 1) den Schluss ziehen: „education is the single most important determinant of occupational success“. Insofern muss a priori bereits in jungen Jahren eingegriffen werden, um die Startbedingungen zu verbessern. In anderen Ländern funktioniert dies offensichtlich besser als in Deutschland und der Schweiz, wo durch die frühe Selektion im Bildungssystem die Weichen für den späteren Erfolg am Arbeitsmarkt, aber auch für die Lebenschancen und -qualität bereits in jungen Jahren gelegt werden. Deswegen muss die Politik in beiden Ländern den Versuch unternehmen, das Bildungsangebot derart zu gestalten, dass die soziale Herkunft weniger bedeutsam wird und auch Kinder aus bildungsfernen Elternhäusern die gleiche Chance auf höhere Bildung haben wie Kinder aus bildungsnahen Schichten. Wenn Kinder aus sozial schwachen bzw. unterprivilegierten Elternhäusern bereits in der frühen Kindheit und Jugend stärker gefördert werden, beispielsweise durch die gezielte Betreuung in Kindergärten, dann verliert die soziale Herkunft nicht nur für den Bildungserfolg sondern auch für den Einkommenserzielungsprozess an Bedeutung.

\section{Literatur}

Aiken, Leona S. / West Stephen G. (1991): Multiple Regression. Testing and Interpreting Interactions, Newbury Park.

Atkinson, Anthony B. (1983): The Economics of Inequality, Oxford.

Becker, Gary S. (1964): Human Capital. A Theoretical and Empirical Analysis with Special Reference to Education, New York u.a.

Bellmann, Lutz / Knut Gerlach / Olaf Hübler (1984): Lohnstruktur in der Bundesrepublik Deutschland. Zur Theorie und Empirie der Arbeitseinkommen, Frankfurt / Main - New York. 
Blau, Peter M. / Otis D. Duncan (1967): The American Occupational Structure, New York u.a.

Borghans, Lex / Angela Lee Duckworth / James J. Heckman / Bas ter Weel (2008): The Economics and Psychology of Personality Traits, in: Journal of Human Resources 43, S. 974-1061.

Boudon, Raymond (1974): Education, Opportunity, and Social Inequality, New York u.a.

Bourdieu, Pierre / Jean-Claude Passeron (1971): Die Illusion der Chancengleichheit. Untersuchungen zur Soziologie des Bildungswesens am Beispiel Frankreichs, Stuttgart.

Bowles, Samuel (1972): Schooling and Inequality from Generation to Generation, in: Journal of Political Economy 80, S. 219-251.

Bowles, Samuel / Herbert Gintis / Melissa Osborne (2001 a): The Determinants of Earnings. A Behavioral Approach, in: Journal of Economic Literature 39, S. 1137-1176.

Bowles, Samuel / Herbert Gintis / Melissa Osborne (2001 b): Incentive-Enhancing Preferences. Personality, Behavior, and Earnings, in: American Economic Review 91, S. 155-158.

Brauns, Hildegard / Susanne Steinmann (1999): Educational Reform in France, West-Germany and the United Kingdom. Updating the CASMIN Educational Classification, in: ZUMA Nachrichten 23, S. 7-44.

Buchmann, Marlis / Maria Charles (1993): The Lifelong Shadow: Social Origins and Educational Opportunities in Switzerland, in: Yossi Shavit / Hans-Peter Blossfeld (Hrsg.), Persistent Inequalities. Changing Educational Stratification in Thirteen Countries, Boulder, S. 177-192.

Buchmann, Marlis / Stefan Sacchi (1998): The Transition from School to Work in Switzerland: Do Characteristics of the Educational System and Class Barriers Matter?, in: Yossi Shavit / Walter Müller (Hrsg.), From School to Work: A Comparative Study of Educational Qualifications and Occupational Destinations, Oxford, S. 407-442.

Davis, Kingsley / Wilbert E. Moore ([1945]1967): Some Principles of Stratification, in: Reinhard Bendix / Seymour M. Lipset (Hrsg.), Class, Status and Power. Social Stratification in Comparative Perspective, London, S. 47-53.

Dustmann, Christian (2004): Parental Background, Primary to Secondary School Transitions and Wages, in: Oxford Economic Papers 56, S. 209-230.

Card, David (1995): Earnings, Schooling, and Ability Revisited, in: Solomon Polachek (Hrsg.), Research in Labor Economics, Vol. 14, Greenwich, S. 23-48.

Erikson, Robert / Jan O. Jonsson (Hrsg.) (1996): Can Education be Equalized? The Swedish Case in Comparative Perspective, Boulder.

Ermisch, John / Marco Francesconi (2001): Family Matters: Impacts of Family Background on Educational Attainment, in: Economica 68, S. 137-156.

Gang, Ira N. / Klaus F. Zimmermann (2000): Is Child Like Parent? Educational Attainment and Ethnic Origin, in: Journal of Human Resources 35, S. 550-569.

Gautschi, Thomas / Dominik Hangartner (2006): Size Does Matter. Körpergröße, Humankapital und Einkommen, in: Soziale Welt 57, S. 273-294.

Girod, Roger (1977): Inégalité, Inégalités: Analyse de la Mobilité Sociale, Paris.

Greene, William H. (2003): Econometric Analysis, Upper Saddle River / NJ.

Hartmann, Michael (2002): Der Mythos von den Leistungseliten: Spitzenkarrieren und soziale Herkunft in Wirtschaft, Politik, Justiz und Wissenschaft, Frankfurt / Main - New York.

Hartmann, Michael / Johannes Kopp (2001): Elitenselektion durch Bildung oder durch Herkunft? Promotion, soziale Herkunft und der Zugang zu Führungspositionen in der deutschen Wirtschaft, in: Kölner Zeitschrift für Soziologie und Sozialpsychologie 53, S. 436-466.

Heckman, James J. / Lance J. Lochner/ Petra E. Todd (2003): Fifty Years of Mincer Earnings Regressions, IZA Discussion Paper No. 775, Bonn: Institute for the Study of Labor (IZA). 
Heineck, Guido (2005): Up in the Skies? The Relationship between Body Height and Earnings in Germany, in: Labour - Review of Labour Economics and Industrial Relations 19, S. 469-489.

Hirschel, Dierk (2004): Einkommensreichtum und seine Ursachen. Die Bestimmungsfaktoren hoher Arbeitseinkommen, Marburg.

Hondrich, Karl O. (1984): Der Wert der Gleichheit und der Bedeutungswandel der Ungleichheit, in: Soziale Welt 35, S. 267-293.

Hradil, Stefan (2001): Soziale Ungleichheit in Deutschland, Opladen.

Jackson, Michelle / John H. Goldthorpe / Colin Mills (2005): Education, Employers, and Class Mobility, in: Research in Social Stratification and Mobility 23, S. 3-33.

Jencks, Christopher (1979): Who gets Ahead? The Determinants of Economic Success in America, New York.

König, Wolfgang / Paul Lüttinger / Walter Müller (1988): A Comparative Analysis of the Development and Structure of Educational Systems. Methodological Foundations and the Construction of a Comparative Educational Scale, CASMIN Working Paper No. 12, Mannheim: Universität Mannheim.

Mincer, Jacob (1976): Progress in Human Capital Analysis of the Distribution of Earnings, in: Anthony B. Atkinson (Hrsg.): The Personal Distribution of Incomes, London, S. 136-192.

Mincer, Jacob (1974): Schooling, Experience, and Earnings, New York - London.

Mincer, Jacob (1970): The Distribution of Labour Incomes: A Survey with Special Reference to the Human Capital Approach, in: Journal of Economic Literature 8, S. 1-26.

Müller, Walter (1972): Bildung und Mobilitätsprozess. Eine Anwendung der Pfadanalyse, in: Zeitschrift für Soziologie 1, S. 65-84.

Müller, Walter / Karl Ulrich Mayer (1976): Chancengleichheit durch Bildung? Untersuchungen über den Zusammenhang von Ausbildungsabschlüssen und Berufsstatus, Gutachten für den Deutschen Bildungsrat 42, Stuttgart.

Müller, Walter / Yossi Shavit (1998): The Institutional Embeddedness of the Stratification Process. A Comparative Study of Qualifications and Occupations in Thirteen Countries, in: Yossi Shavit / Walter Müller (Hrsg.), From School to Work. A Comparative Study of Educational Qualifications and Occupational Destinations, Oxford, S. 1-48.

Mulligan, Casey B. (1999): Galton vs. the Human Capital Approach to Inheritance, in: Journal of Political Economy 107, S. 184-224.

Nyhus, Ellen K. / Empar Pons (2005): The Effects of Personality on Earnings. in: Journal of Economic Psychology 26, S. 363-384.

OECD (2004): Education at a Glance 2004, Paris.

Prenzel, Manfred / Jürgen Baumert / Werner Blum / Rainer Lehmann / Detlev Leutner / Michael Neubrand / Reinhard Pekrun / Hans-Günter Rolff / Jürgen Rost / Ulrich Schiefele (Hrsg.) (2004): PISA 2003. Der Bildungsstand der Jugendlichen in Deutschland - Ergebnisse des zweiten internationalen Vergleichs, Münster u.a.

Ramseier, Erich / Christian Brühwiler (2003): Herkunft, Leistung und Bildungschancen im gegliederten Schulsystem: Vertiefte PISA-Analyse unter Einbezug der kognitiven Grundfähigkeiten, in: Schweizerische Zeitschrift für Bildungswissenschaften 25, S. 23-57.

Saterdag, Hermann / Heinz Stegmann (1980): Determinanten von Bildungsentscheidungen, in: Ulrich Beck / Karl H. Hörning / Wilke Thomssen (Hrsg.), Bildungsexpansion und betriebliche Beschäftigungspolitik. Aktuelle Entwicklungstendenzen im Vermittlungszusammenhang von Bildung und Beschäftigung, Beiträge zum 19. Deutschen Soziologentag, Berlin 1979, Frankfurt / Main - New York, S. $115-130$.

Schimpl-Neimanns, Bernhard (2000): Soziale Herkunft und Bildungsbeteiligung. Empirische Analysen zu herkunftsspezifischen Bildungsungleichheiten zwischen 1950 und 1989, in: Kölner Zeitschrift für Soziologie und Sozialpsychologie 52, S. 636-669. 
Schnapp, Kai-Uwe (1997): Soziodemographische Merkmale der bundesdeutschen Eliten, in: Wilhelm Bürklin / Hilke Rebenstorf (Hrsg.), Eliten in Deutschland. Rekrutierung und Integration, Opladen, S. 69-99.

Schneider, Thorsten (2004): Hauptschule, Realschule oder Gymnasium? Soziale Herkunft als Determinante der Schulwahl, in: Marc Szydlik (Hrsg.), Generation und Ungleichheit, Wiesbaden, S. 77-103.

Schwarze, Johannes (1991): Ausbildung und Einkommen von Männern - Einkommensfunktionsschätzungen für die ehemalige DDR und die Bundesrepublik Deutschland, in: Mitteilungen aus der Arbeitsmarkt- und Berufsforschung (MittAB) 24, S. 63-69.

Sewell, William H. / Archibald O. Haller / Alejandro Portes (1969): The Educational and Early Occupational Attainment Process, in: American Sociological Review 34, S. 82-92.

Shavit, Yossi / Hans-Peter Blossfeld (Hrsg.) (1993): Persisting Inequality - Changing Educational Attainment in Thirteen Countries, Boulder u.a.

SOEP Group (2001): The German Socio-Economic Panel (GSOEP) After More Than 15 Years - Overview, in: Elke Holst / Dean R. Lillard / Thomas A. DiPrete (Hrsg.), Proceedings of the 2000 Fourth International Conference of German Socio-Economic Panel Study Users (GSOEP 2000), in: Vierteljahreshefte zur Wirtschaftsforschung 70, Berlin, S. 7-14.

Szydlik, Marc (1993): Arbeitseinkommen und Arbeitsstrukturen. Eine Analyse für die Bundesrepublik Deutschland und die Deutsche Demokratische Republik, Berlin.

Treiman, Donald J. (1977): Occupational Prestige in Comparative Perspective, New York.

Wolff, Edward N. (1997): Economics of Poverty, Inequality, and Discrimination, Cincinnati / OH.

Zimmermann, Erwin / Monica Budowski (in Zusammenarbeit mit Alexis Gabadinho / Annette Scherpenzeel / Robin Tillmann / Boris Wernli) (2003): The Swiss Household Panel Survey. A Multidimensional Database for Monitoring Social Change, in: Dominique Joye / Isabelle Renschler / François Hainard (Hrsg.), Social Change and Social Reporting, Neuchâtel: UNESCO, SIDOS, S. 137-156.

Bettina Isengard Universität Zürich Soziologisches Institut Andreasstrasse 15

CH-8050 Zürich isengard@soziolgie.uzh.ch 